\title{
Histopathologic Finding of Soft Tissue Associated with Impacted Mandibular Third Molars that Did Not Exhibit Pathologic Pericoronal Radiolucency \\ A Tak ${ }^{1}, \mathrm{R} \mathrm{Metgud}^{2}, \mathrm{M}$ Astekar ${ }^{3}, \mathrm{M} \mathrm{Tak}^{4}$
}

\begin{abstract}
Objectives: The present study was executed with the objective to evaluate the histopathological features of pericoronal follicles (PFs) of asymptomatic impacted mandibular third molars that did not exhibit pathologic pericoronal radiolucency.
\end{abstract}

Study design: A cross-sectional study was conducted among pericoronal follicular specimen of soft tissue associated with asymptomatic, radiographically normal impacted mandibular third molars from 95 subjects. Intraoral periapical radiographs (IOPA) and orthopantomogram of all subjects were recorded and traced using tracing paper. After the tooth removal, the follicle was carefully enucleated from the socket attachment. The cut sections were stained with Haematoxylin and eosin stain and sections were microscopically evaluated. Epithelial cell proliferation was determined by immunohistochemical labelling. The associations between the attributes were tested using the Chi-Square Test.

Results: Thirty seven [37.3\%] specimen revealed dentigerous cyst-like changes and two [2.1\%] specimens revealed odontogenic keratocyst [OKC]-like changes. Immunohistochemical evaluation of proliferation marker Ki-67 was found to be positive in only $7.69 \%$ of the hyperplastic type of epithelium.

Conclusion: The data revealed that there is a risk of pathologic changes associated with radiographically normal asymptomatic impacted mandibular third molar.

Keywords: Cell proliferation activity, dental follicle, impaction, pathologic changes

From: ${ }^{1}$ Department of Oral Pathology and Microbiology, Pacific Dental College and Hospital, Airport Road, Debari, Udaipur, Rajasthan, India. Pin: 313024 and ${ }^{2}$ Department of Oral Pathology and Microbiology, Institute of Dental Sciences, Bareilly, Uttar Pradesh, India.

Correspondence: Dr A Tak, Department of Oral Pathology and Microbiology, Pacific Dental College and Hospital, Debari, Airport Road, Udaipur, Rajasthan, India. 


\section{INTRODUCTION}

Research now indicates that the reasons for most third molar problems today are not due to evolutionary changes but other reasons $(1,2)$. The removal of third molar is one such common practice in dentistry where in the decision making on whether and when to extract has been a subject of constant debate. Although, there are therapeutic and prophylactic indications for the removal of impacted third molar, with no general agreement about the need for surgical removal of all asymptomatic impacted third molars (3).

Unfortunately, some problems associated with wisdom teeth such as cysts and tumors may be impossible to predict. The dental follicular tissues frequently have been confused with odontogenic tumors by both medical pathologists and clinicians. Histomorphologically, some odontogenic fibromas and myxomas, which are thought to originate from mesenchymal odontogenic tissues, are markedly similar to dental follicular and dental papillary tissues. Correlation of the clinical, radiographic, and histopathological findings is often necessary to establish a proper diagnosis and to avoid unnecessary surgical procedures (4).

$\mathrm{Ki}-67$ has been used to investigate the proliferation potential of normal tissues, as well as preneoplastic and neoplastic lesions. The identification of abnormally increased proliferation of precursor cells is an important aspect of neoplastic progression (5). Ki-67 immunohistochemical staining using proliferation markers, particularly MIB-1, a monoclonal antibody to the Ki-67 antigen, is useful for objective histopathological evaluation of tumour proliferation activity (6).

In the majority of the cases, the presence of cyst and tumours are not confirmed at early stages, which can become hazardous in latter stages of life. However, not all pericoronal cysts and tumours are obvious clinically and radiographically. Hence the present study was planned to evaluate histopathological features of pericoronal follicles [PFs] of 
asymptomatic impacted mandibular third molars that did not exhibit pathologic pericoronal radiolucency.

\section{SUBJECTS AND METHOD}

Study design and study area: A cross sectional study was conducted among subjects with radiographically normal, asymptomatic, impacted mandibular third molars in the Department of Oral Pathology and Microbiology, Pacific Dental College and Hospital, Udaipur, Rajasthan.

Study population: The study group comprised specimens of pericoronal follicles from 95 subjects having at least one impacted mandibular third molar tooth without obvious clinical and radiographic features.

\section{Inclusion criteria}

Mandibular impacted third molar.

Patients advised for removal of mandibular third molars as per orthodontic needs, prosthetic reasons or having an angulation unfavourable for eruption, etc.

Subjects with radiographic follicular space within normal limits $[<2.5 \mathrm{~mm}]$.

\section{Exclusion criteria}

Patients complaining of any clinical symptoms and obvious pathologies associated with impacted mandibular third molars.

Patients with any systemic diseases.

The study protocol was reviewed by the Ethics Committee of Pacific Dental College and Hospital and was granted ethical clearance. Official permission to conduct the study was obtained from the Principal of Pacific Dental College and Hospital, Udaipur. 
After explaining the purpose and details of the study, a written informed consent was obtained from all the study subjects.

\section{METHOD}

Sample Selection and sample size: Detailed case history of subjects referred for prophylactic removal of impacted mandibular third molars to the Department of Oral \& Maxillofacial Surgery, Pacific Dental College and Hospital from April 2011 to April 2012 was recorded. Subjects without the obvious clinical and radiographic features were selected. The final sample size of 95 subjects was thus achieved.

Radiographic evaluation: Intraoral periapical radiographs (IOPA) and orthopantomogram (OPG) of the asymptomatic impacted tooth of all subjects were obtained and evaluated. The contours of the tooth and of the pericoronal space were traced on tracing paper using the Xray viewer. The widest point of the follicular radiolucency was measured using a graduated scale. Minimal distortion of the radiograph was not considered while measuring.

The impaction was classified according to Pell and Gregory classification.

The surgical procedure was carried out under local anaesthesia with all aseptic measures with Ward's third molar incision. The coronal follicular tissue was carefully dissected using blunt forceps and preserved. After the tooth was removed, the follicle was carefully enucleated from the socket attachment. The surgical site was irrigated and closed with 3-0 silk sutures.

\section{Histopathological technique}

The excised specimen was immediately washed and spread on Watman's number one filter paper to make it flat and the paper was folded without folding the specimen. The specimen was fixed in $10 \%$ neutral buffered formalin, processed, impregnated in paraffin wax and 5 
$\mu \mathrm{m}$ thick sections were prepared from paraffin blocks and stained with hematoxylin and eosin stain.

\section{Staining procedure}

Formalin fixed paraffin embedded tissues were sectioned at $5 \mu \mathrm{m}$, using a semi-automatic microtome. Consecutive sections were placed on slides pre-coated with egg albumin for routine haematoxylin and eosin staining.

The staining protocols followed in the present study were as follows:

The Standard Haematoxylin and Eosin Staining Protocol [Regressive].

The Immunohistochemical Staining Protocol for Ki-67 protein.

The evaluation of the stained slides was carried out using a binocular research microscope [Lawrence \& Mayo] under 100X and 400X magnification mounted with digital camera. Three different Oral Pathologists reviewed and evaluated the stained microscopic slides. The same slide was read on microscope independently by each pathologist, and each was unaware of the diagnoses of the others. To standardize histologic assessment of soft tissue specimens, the participating pathologists developed a consensus definition of 'dentigerous cyst'. They agreed that any soft tissue specimen with stratified squamous epithelium equal to or more than 4-5 cell layers thick spreading along the surface of the follicle with dense fibrous connective tissue stroma would be deemed cystic. Other pathological findings were reported according to accepted diagnostic criteria.

The features evaluated by the Oral Pathologists were: Epithelial tissue: Present [reduced enamel epithelium/stratified squamous epithelium] or absent; Epithelial cell activity: Inactive or hyperplastic; Connective tissue type: Fibrous, Myxomatous or Both; presence or absence of inflammation; presence or absence of epithelial odontogenic rests; Pathological changes: cystic and neoplastic changes. 


\section{Determination of KI-67 Expression}

The expression of Ki-67 was determined with respect to Localization, Area [percentage) and Intensity of stained cells in the epithelium as specified by Vered et al [2009] (7) and Jahanshahi et al [2006] (8).

The observations were tabulated and the associations between the attributes were tested using the Chi-Square Test. The software used for statistical analysis was Statistical Package for Social Sciences version 19 [SPSS, Chicago, IL, USA] $p$ - value was set at $\leq 0.05$.

\section{RESULTS}

The age range of the study population was 15 to 55 years, with mean age of 27.9 years.

Microscopically, 59 [62.2\%] demonstrated presence of epithelium and one specimen was insufficient for any interpretation. Stratified squamous epithelium [SSE] and reduced enamel epithelium [REE] [Fig.1] were observed among 39 [41.1\%] and 20 [21.1\%] specimens, respectively. Hyperplastic epithelial cell activity was observed in 37 [38.9\%] specimens. Connective tissue was observed to be fibrous in 78 [82.1\%] and myxoid in 9 [9.5\%] specimens, however 7 [7.4\%] specimens revealed presence of both. Among all, chronic inflammation and odontogenic epithelial rests were observed among 59 [62.1\%] and 38 [40\%] specimens respectively [Table 1).

Of the 95 specimens submitted for histopathologic examination, 39 [39.0\%] specimens were deemed to have cystic changes. Among them, 37 [37.3\%] specimens revealed dentigerous cyst like changes [Fig.2] and 2 [2.1\%] specimens revealed odontogenic keratocyst [OKC] like changes (Fig. 3).

When the epithelium was correlated with inflammation, statistically significant $[p=$ 0.000] results were obtained. The presence of inflammation was observed among 29 [74.4\%], 
$3[15 \%]$ and 27 [77.1\%] specimens with stratified squamous epithelium, reduced enamel epithelium and specimens without epithelium respectively (Table 2).

On correlation of age with different histopathological criteria, statistically significant results were obtained with epithelium $[p=0.000]$, and cell activity $[p=0.005]$. The proportion of REE was highest among the 15-25 years age group and SSE in the $>45$ years age group. Age correlation with cell activity revealed significant increase in hyperplastic epithelium with increase in age (Table 3).

On correlation of positions of third molar in relation to long axis of $2^{\text {nd }}$ molar with different histopathological criteria, no significant results were obtained with epithelium, cell activity, inflammation and odontogenic rests. Type of connective tissue revealed a statistically significant difference with position of third molar in relation to long axis of $2^{\text {nd }}$ $\operatorname{molar}[p=0.002]$.

Immunohistochemistry revealed only 3 [7.69\%] pericoronal follicles with positive staining for Ki-67 [Fig. 4], out of which one follicular specimen revealed uniform staining in the basal and suprabasal layers and two showed focal immunopositivity. Data on correlations of epithelium and connective tissue criteria with gender presented non- significant results and hence are not reported.

\section{DISCUSSION}

The commonest reason for partial eruption or complete impaction of third molar is decreased size of the jaw, which results in lack of space for eruption. After the formation of enamel, the crown of the tooth is surrounded by reduced enamel epithelium and by ectomesenchyme. These two structures form the dental follicle, which can be the origin of several types of diseases during or after odontogenesis (9). Still the prophylactic removal of impacted third 
molar remains a controversy. The present study was carried out to determine the pathologic changes associated with asymptomatic impacted third molars, because these may result in a health risk to the subjects.

Absence of epithelium was observed in $36.8 \%$ of the specimens. Kotrashetti et al (11) found $12.19 \%$ of the cases which were devoid of epithelium, Damante and Fleury (9) found 13\% cases, and Khorasani and Samiezadeh (12) reported 31\% cases. Damante and Fleury (9) concluded that the loss of epithelium may have resulted from the ameloblast cell adherence to the enamel cuticle, which detaches from parts of the specimens during surgical treatment.

Theoretically, inflammation should not be expected in follicles of asymptomatic impacted teeth. Inflammation was associated with stratified squamous epithelium in $74.4 \%$. Khorasani and Samiezadeh (12) found that inflammation was significantly associated with squamous metaplasia which was in accordance with our study. The percentage of specimen with inflammation in the present study were higher than those by Kotrashetti et al (11). The presence of inflammation can be explained by two hypotheses: the first one is physiological.

The erupting process occurs with an inflammation originating from the penetration of oral antigens into the wider intercellular spaces of the epithelial cells of the reduced enamel organ and oral epithelium. Many teeth could be erupting, although this process would not be completed. The second hypothesis is that many unerupted teeth may communicate with the oral environment through the periodontal pocket of the adjacent tooth (9). In the present study, follicular tissue with the impacted third molar was deemed cystic if lined by stratified squamous epithelium. This finding is consistent with the studies conducted by Curran et al (13). It seems that the longer the follicular tissues remain in the bone, the larger the possibility of an inflammation. Various factors including a previous periodontal abscess, second molar periodontitis or physiologic alveolar bone resorption may be responsible for this event. Also with increasing age, bone resorption occurs which gradually exposes the third molar to the oral cavity and subsequently to increased risk of infection (12). 
In the present study, 39\% of the follicles revealed cystic changes, Rakprasitkul (14) and Yildirim et al (10) observed 58.6\%, and 23\% cases with pathologic changes in there studies respectively Curran et al (13) demonstrated 77.5\% dentigerous cyst, 9.1\% OKC and 1.5\% ameloblastoma. Mesgarzadeh et al (15) demonstrated dentigerous cyst $38 \%$ and ameloblastic changes $5.8 \%$ and Kotrashetti et al (11) demonstrated $51.2 \%$ cystic changes, $4.8 \%$ odontogenic keratocyst and $2.4 \%$ ameloblastoma like proliferation. In a study conducted by Mehrotra and David [2010] (16), 90\% of the impacted third molars associated with cystic changes were the mandibular impacted third molars, which is in agreement with the findings of Glosser and Campbell (17) who stated that the mandibular impacted third molar to be associated with highest incidence of cystic changes. Spontaneous cyst involution occurring over a period of time, or conversion of the tissue to a quiescent state, could result in a histological aberration with little clinical significance (18).

In the present study, on correlation of age with different histopathological criterias, it was observed that the incidence of squamous epithelium significantly increased with age. These findings were in accordance to Khorasani and Samiezadeh [2008] (12). This increase in squamous metaplasia with advancing age may be due to factors such as chronic inflammation or other age-induced effects on dental follicular tissues. This squamous metaplasia might be considered an adaptive reaction in cells vulnerable to stress, improving their tolerance to unsuitable environmental conditions. (12).

Girod et al (19) reported that the development of large cysts around impacted third molars took 2-13 years. It seems, therefore that the longer an impaction exists, the greater the risk of development of cysts and tumours. Increase in cell activity with development may be related to the increase in inflammation (20). De Paula et al (21) suggested that chronic inflammation may cause chronic irritation and stimulate the proliferation of epithelial cells. In the acutely mesioangularly impacted tooth, therefore, food is forced into the space between 
the distal surface of the second molar and the occlusal surface of the third molar. Conversely, in vertical, distoangular and less acute mesioangular impactions (22),

Presence of odontogenic epithelial rests in connective tissue was reported in $40.4 \%$ of the specimens which was higher than found in the study by Kotrashetti et al (11) but lesser than Khorasani and Samiezadeh (12) [48\%], Kim and Ellis (6) [79\%], and Conklin and Stafne [1949](23) [86\%].

We observed that stratified squamous epithelium, hyperplastic cell activity, fibrous connective tissue, inflammation, and odontogenic rests was more in class 1 impaction. On literature review no studies on comparison of position of third molars in relation to ramus of mandible with these criteria were revealed.

In the present study, when position of the third molar in relation to the long axis of the $2^{\text {nd }}$ molar with different histopathological criteria were correlated, vertical and distoangularly inclined molars showed a greater tendency towards pathological changes followed by mesioangular and horizontal. The results were statistically significant when compared with connective tissue type. The higher probability of cystic changes was found in the vertically positioned impacted third molar, in a study by Baykul et al (24) which is in accordance with our study but Eliasson et al (25) reported a higher incidence of pathological changes in horizontal impacted third molars in their radiographic study which is in contrast to our study.

In the present study, 39 specimens with stratified squamous epithelium when immunostained with Ki-67 antibody revealed positive staining with 3 [7.69\%] specimens, out of which one specimen revealed uniform staining in basal and suprabasal layer and two specimens revealed focal immunopositivity. Though the study is being performed with caution, one of the limitation of the present study is small sample size. Hence, further studies with an amplified sample size are recommended. 


\section{CONCLUSION}

The overall results gained from the current study indicated that clinical judgement based solely on radiographic appearance may be quite deceptive. According to the present study, cystic changes can develop in clinically asymptomatic impacted third molars. The data from this study although cannot justify the complement removal of all asymptomatic impacted third molars but suggest that there is a risk of pathologic changes. Thus, the extraction of the third molar should be carried out near the age of 25 seeing the position and condition of $2^{\text {nd }}$ and $3^{\text {rd }}$ molar. 


\section{REFERENCES}

1. Bergman J. Are wisdom teeth [third molars] vestiges of human evolution? Technical J 1998; 12: 297-304.

2. Farish SE, Bouloux GF. General technique of third molar removal. Oral Maxillofac Surg Clin N Am 2007; 19: 23-43.

3. Shetty DC, Ahuja P, Urs AB, Bablani D, Paul M. Epidemiological status of 3rd molars - their clinical implications. J Oral Health Comm Dent 2010; 4: 12-15.

4. Kim J, Ellis GL. Dental follicular tissue: misinterpretation as odontogenic tumors. J Oral Maxillofac Surg 1993; 51: 762-7.

5. Kucukkolbasi H, Esen A. KI67 expression in dental follicle of the impacted teeth: A case report. SÜ Dişhek Fak Derg 2012; 20: 63-6.

6. Hasegawa T. Standardization of Ki-67 Immunohistochemical Staining for Diagnosing Grade of Gastrointestinal Stromal Tumor [GIST]. Connection 2008; 50-4.

7. Vered M, Allon I, Dayan D. Maspin, p53, p63, and Ki-67 in epithelial lesions of the tongue: from hyperplasia through dysplasia to carcinoma. J Oral Pathol Med 2009; 38: $314-20$.

8. Jahanshahi G, Talebi A, Shirvani A. Expression of bcl-2 in the Epithelial Lining of Odontogenic Keratocysts. J Dent [Tehran] 2006; 3: 30-5.

9. Damante JH, Fleury RN. A contribution to the diagnosis of the small dentigerous cyst or the paradental cyst. Pesqui Odontol Bras 2001; 15: 238-46.

10. Yildirim G, Ataoğlu H, Mihmanli A, Kiziloğlu D, Avunduk MC. Pathologic changes in soft tissues associated with asymptomatic impacted third molars. Oral Surg Oral Med Oral Pathol Oral Radiol Endod 2008; 106: 838-42. 
11. Kotrashetti VS, Kale AD, Bhalaerao SS, Hallikeremath SR. Histological changes in soft tissue associated with radiographic normal impacted 3rd molar. Indian J Dent Res 2010; 21: 285-90.

12. Khorasani M, Samiezadeh F. Histopathologic evaluation of follicular tissues associated with impacted third molars. J Dent [Tehran] 2008; 5: 65-70.

13. Curran AE, Damm DD, Drummond JF. Pathologically significant pericoronal lesions in adults: histopathologic evaluation. J Oral Maxillofac Surg 2002; 60: 613-7.

14. Rakprasitkul S. Pathological changes in the pericoronal tissues of unerupted third molars. Quintessence Int 2001; 32: 233-38.

15. Mesgarzadeh AH, Esmailzadeh H, Abdolrahimi M, Shahamfar M. Pathosis associated with radiographically normal follicular tissues in third molar impactions: A clinicopathological study. Indian J Dent Res 2008; 19: 208-12.

16. Mehrotra V, David MP. Pathologic changes in dental follicle associated with third molar impactions - a study. Pakistan Oral and Dent J 2010; 30: 1-6.

17. Glosser JW, Campbell JH. Pathologic change in soft tissue associated with radiographically 'normal' third molar impactions. Br J Oral Maxillofac Surg 1999; 37: $259-60$.

18. Adelsperger J, Campbell JH, Coates DB, Summerlin DJ, Tomich CE. Early soft tissue pathosis associated with impacted third molars without pericoronal radiolucency. Oral Surg. Oral Med. Oral Pathol Oral Radiol Endod 2000; 89: 402-6.

19. Girod SC, Gerlach KL, Kruhger G. Cysts associated with longstanding impacted third molars. Int J Oral Maxillofac Surg 1993; 22: 110-2.

20. Simşek-Kaya G, Özbek E, Kalkan Y, Yapici G, Dayi E, Demirci T. Soft tissue pathosis associated with asymptomatic impacted lower third molars. Med Oral Patol Oral Cir Bucal 2011; 16: e924-31. 
21. De Paula AM, Carvalhais JN, Domingues MG, Barreto DC, Mesquita RA. Cell proliferation markers in the odontogenic keratocyst: effect of inflammation. J Oral Pathol Med 2000; 29: 477-82.

22. Colgan CM, Henry J, Napier SS, Cowan CG. Paradental cysts a role for food impaction in the pathogenesis. British J Oral Maxillofac Surg 2002; 40: 163-8.

23. Conklin WW, Stafne EC. A study of odontogenic epithelium in the dental follicle. J Am Dent Assoc 1949; 39: 143-8.

24. Baykul T, Saglam AA, Aydin U, Başak K. Incidence of cystic changes in radiographically normal impacted lower third molar follicles. Oral Surg Oral Med Oral Pathol Oral Radiol Endod 2005; 99: 542-5.

25. Eliasson S, Heimdahl A, Nordenram A. Pathological changes related to long-term impaction of third molars. A radiographic study. Int J Oral Maxillofac Surg 1989; 18: $210-2$. 
Table 1: Histological observations in the pericoronal follicles

\begin{tabular}{lll}
\hline Tissue Characteristics & Number & \% \\
\hline Epithelium & 35 & 36.8 \\
Absent & 20 & 21.1 \\
REE & 39 & 41.1 \\
SSE & & \\
Epithelial cell activity & 20 & 21.1 \\
Inactive & 37 & 38.9 \\
Hyperplastic & & \\
Connective tissue & 9 & 9.5 \\
Myxoid & 78 & 82.1 \\
Fibrous & 7 & 7.4 \\
Mixed & & \\
Inflammation & 35 & 36.8 \\
Absent & 59 & 62.1 \\
Present & & \\
Odontogenic rests & 56 & 58.9 \\
Absent & 38 & 40 \\
Present & 1 & 1.1 \\
Insufficient tissue & 95 & 100 \\
Total & & \\
\hline
\end{tabular}

Table 2: Correlation of epithelium in relation to inflammation

\begin{tabular}{|c|c|c|c|c|c|c|c|}
\hline \multirow{2}{*}{ Epithelium } & \multicolumn{4}{|c|}{ Inflammation } & \multicolumn{2}{|l|}{ Absent } & \multirow{2}{*}{ p-value } \\
\hline & Number & $\%$ & Number & $\%$ & Number & $\%$ & \\
\hline Absent & 35 & 37.2 & 27 & 77.1 & 8 & 22.9 & \multirow{4}{*}{$\begin{array}{l}0.000 \\
{[\mathrm{~S}]}\end{array}$} \\
\hline REE & 20 & 21.3 & 3 & 15 & 17 & 85 & \\
\hline SSE & 39 & 41.5 & 29 & 74.4 & 10 & 25.6 & \\
\hline Total & 94 & 98.9 & 59 & 62.8 & 35 & 37.2 & \\
\hline
\end{tabular}


Table 3: Correlation of age with different histopathological criteria

\begin{tabular}{|c|c|c|c|c|c|c|c|c|c|c|c|c|c|c|c|c|c|c|c|c|c|c|c|c|c|c|c|c|c|}
\hline \multirow{4}{*}{$\begin{array}{l}\text { Age } \\
\text { [yrs] }\end{array}$} & \multicolumn{12}{|c|}{ Epithelium } & \multicolumn{17}{|c|}{ Connective tissue } \\
\hline & \multicolumn{6}{|c|}{ Type } & \multicolumn{6}{|c|}{ Cell activity } & \multicolumn{7}{|c|}{ Types } & \multicolumn{5}{|c|}{ Inflammation } & \multicolumn{5}{|c|}{ Odontogenic Rests } \\
\hline & \multicolumn{2}{|c|}{ Absent } & \multicolumn{2}{|c|}{ REE } & \multicolumn{2}{|c|}{ SSE } & \multirow{2}{*}{$\begin{array}{l}\text { p- } \\
\text { value }\end{array}$} & \multicolumn{2}{|c|}{ Inactive } & \multicolumn{2}{|c|}{ Hyperplastic } & \multirow[t]{2}{*}{$\begin{array}{l}\text { p- } \\
\text { value }\end{array}$} & \multicolumn{2}{|c|}{ Myxoid } & \multicolumn{2}{|c|}{ Fibrous } & \multicolumn{2}{|c|}{ Mixed } & \multirow[t]{2}{*}{$\begin{array}{l}\text { p- } \\
\text { value }\end{array}$} & \multicolumn{2}{|c|}{ Absent } & \multicolumn{2}{|c|}{ Present } & \multirow[t]{2}{*}{$\begin{array}{l}\text { p- } \\
\text { value }\end{array}$} & \multicolumn{2}{|c|}{ Absent } & \multicolumn{2}{|c|}{ Present } & \multirow[t]{2}{*}{$\begin{array}{l}\text { p- } \\
\text { value }\end{array}$} \\
\hline & No. & $\%$ & No. & $\%$ & No. & $\%$ & & No. & $\%$ & No. & $\%$ & & No. & $\%$ & No. & $\%$ & No. & $\%$ & & No. & $\%$ & No. & $\%$ & & No. & $\%$ & No. & $\%$ & \\
\hline $\begin{array}{l}15- \\
25\end{array}$ & 27 & 58.7 & 12 & 26.1 & 7 & 15.2 & \multirow{4}{*}{$0.000^{*}$} & 12 & 70.1 & 5 & 29.4 & \multirow{4}{*}{$0.005^{*}$} & 8 & 17.4 & 32 & 69.6 & 6 & 13 & \multirow{4}{*}{0.064} & 21 & 45.7 & 25 & 54.3 & & 28 & 60.9 & 18 & 39.1 & \multirow{5}{*}{0.503} \\
\hline $\begin{array}{l}26- \\
35\end{array}$ & 7 & 19.4 & 7 & 19.4 & 22 & 61.1 & & 7 & 24.1 & 22 & 75.9 & & 1 & 2.8 & 34 & 94.4 & 1 & 2.8 & & 10 & 27.8 & 26 & 72.2 & & 19 & 52.8 & 17 & 47.2 & \\
\hline $\begin{array}{l}36- \\
45\end{array}$ & 1 & 12.5 & 1 & 12.5 & 6 & 75 & & 1 & 14.3 & 6 & 85.7 & & 0 & 0 & 8 & 100 & 0 & 0 & & 4 & 50 & 4 & 50 & 0.25 & 6 & 75 & 2 & 25 & \\
\hline$>45$ & 0 & 0 & 0 & 0 & 4 & 100 & & 0 & 0 & 4 & 100 & & 0 & 0 & 4 & 100 & 0 & 0 & & 0 & 0 & 4 & 100 & & 3 & 75 & 1 & 25 & \\
\hline Total & 35 & 37.2 & 20 & 21.3 & 39 & 41.5 & & 20 & 35.1 & 37 & 64.9 & & 9 & 9.6 & 78 & 82.9 & 7 & 7.4 & & 35 & 37.2 & 59 & 62.8 & & 56 & 59.6 & 38 & 40.4 & \\
\hline
\end{tabular}




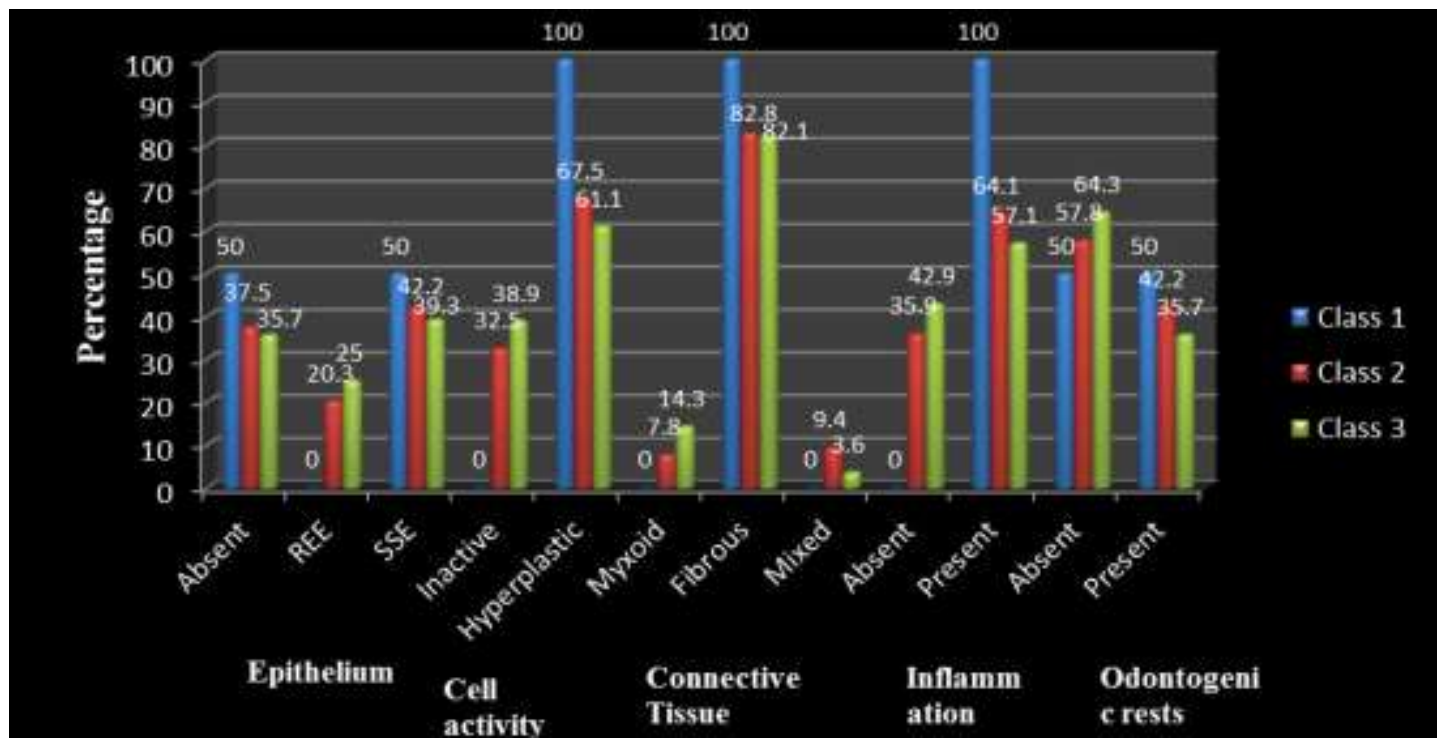

Fig. 1: Correlation of radiographic position of third molar in relation to ramus of mandible with different histopathological criteria.

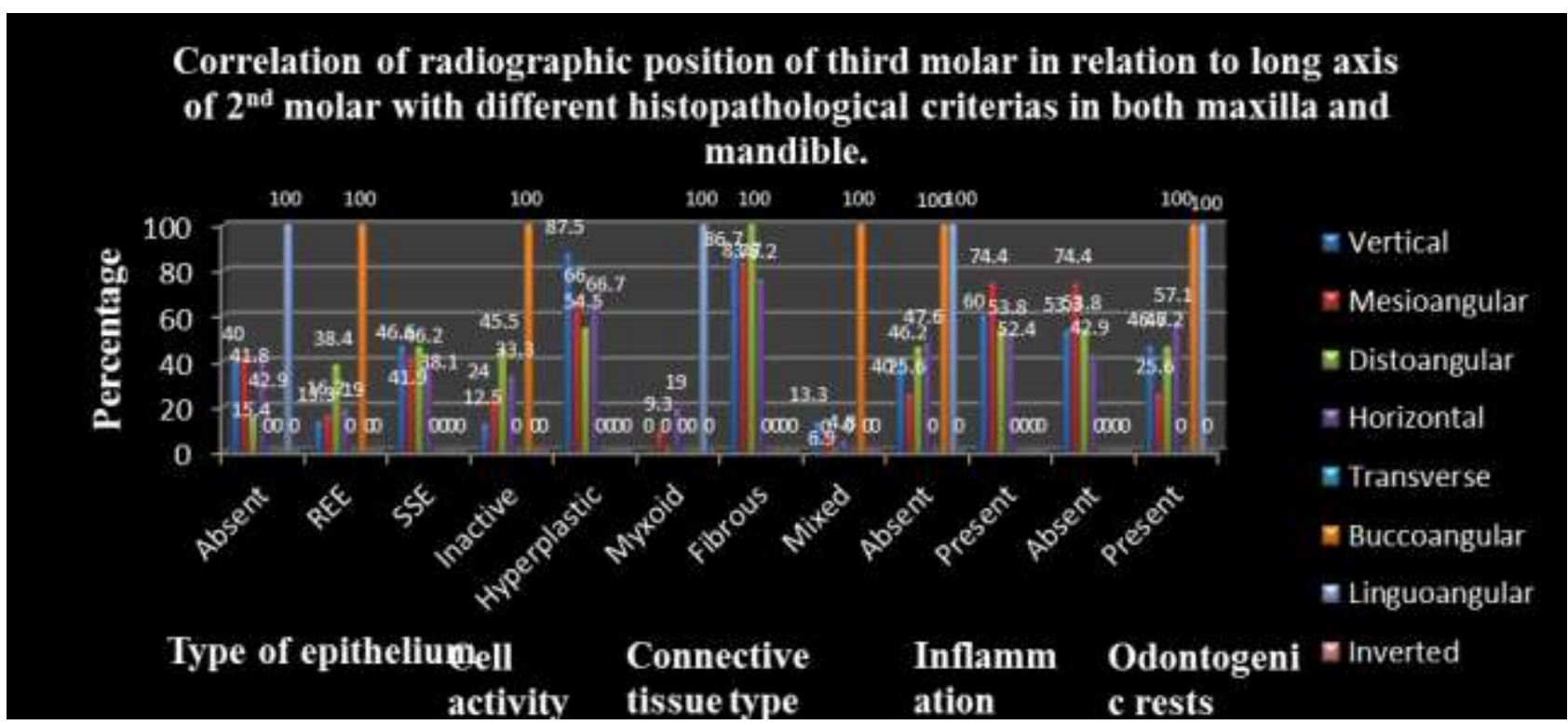

Fig. 2: Correlation of radiographic position of third molar in relation to long axis of $2^{\text {nd }}$ molar with different Histopathological criteria. 


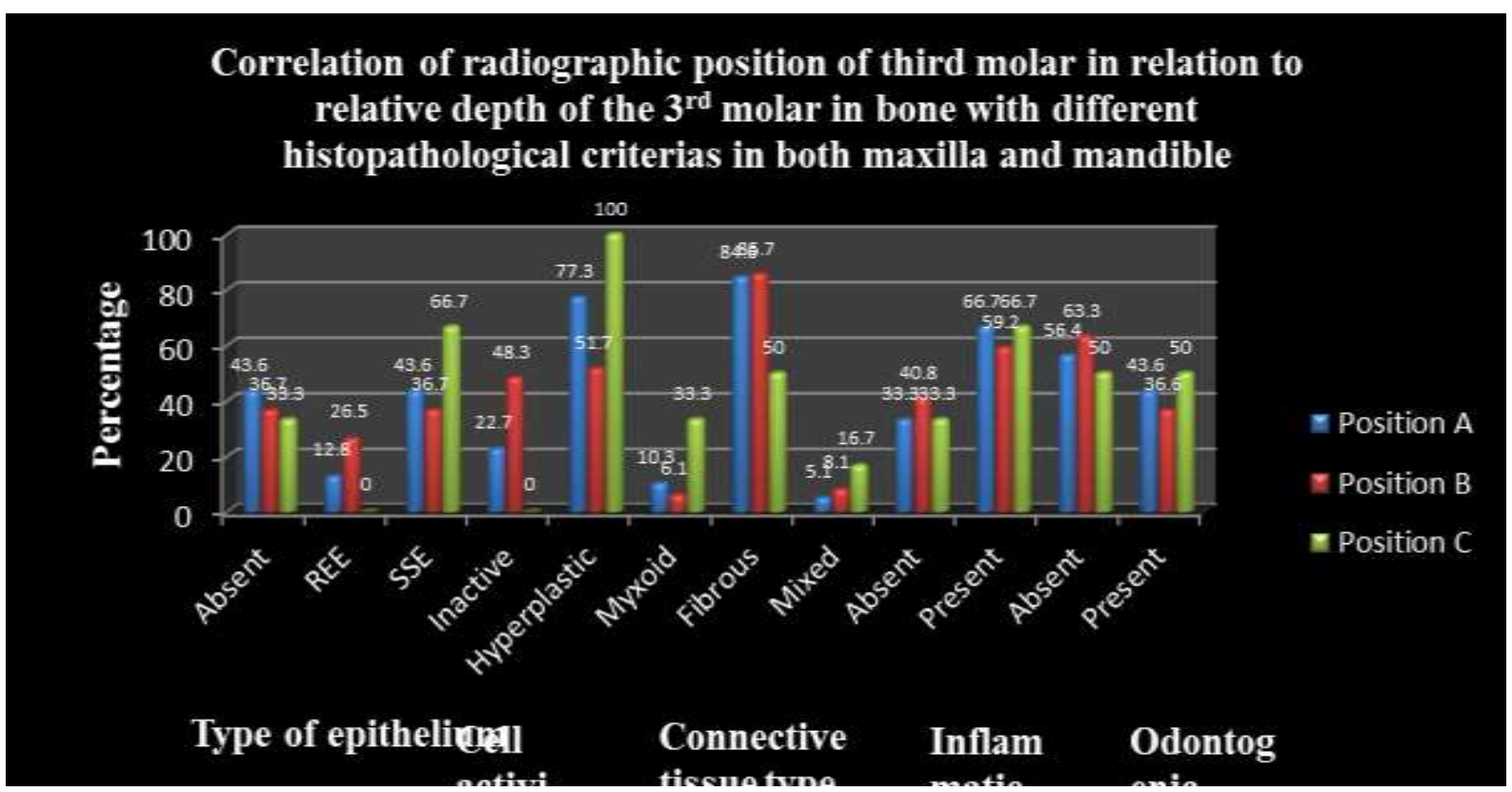

Fig. 3: Correlation of radiographic position of third molar in relation to relative depth of the $3^{\text {rd }}$ molar in bone with different histopathological criteria.

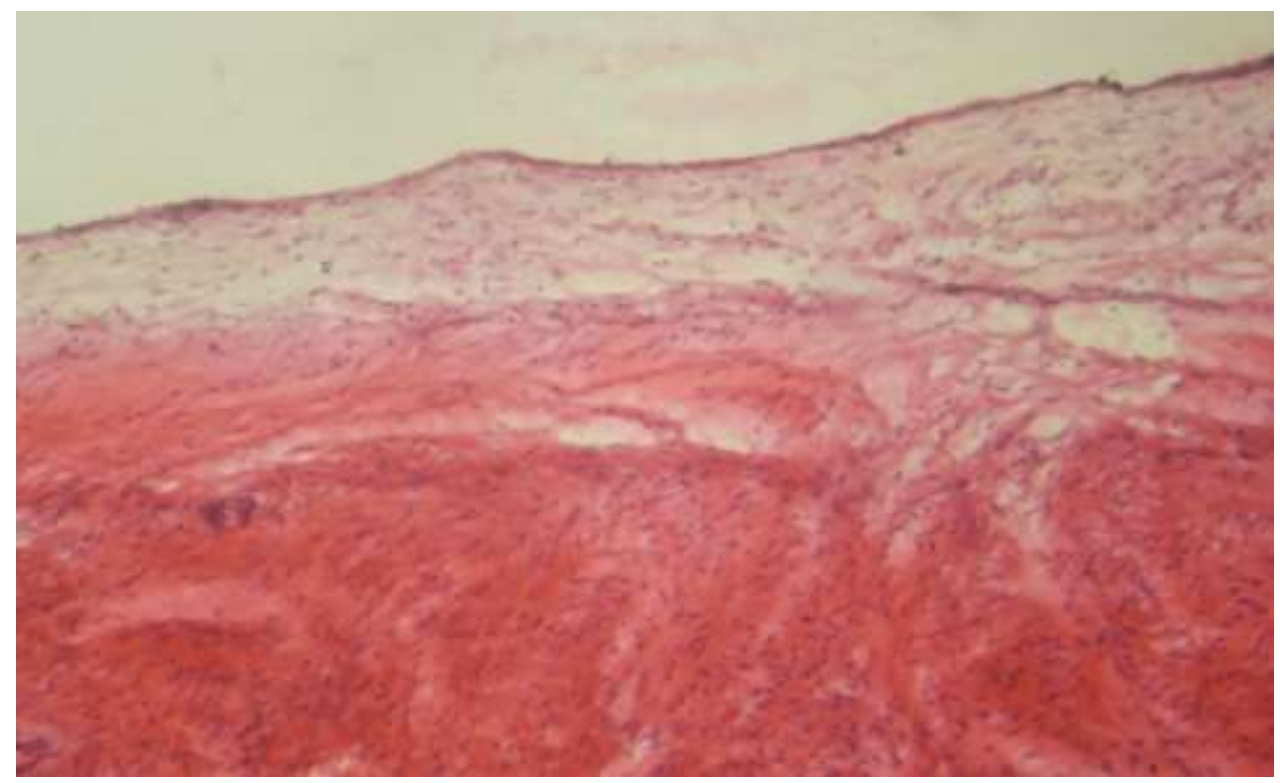

Fig. 4: Reduced enamel epithelium 10X. 


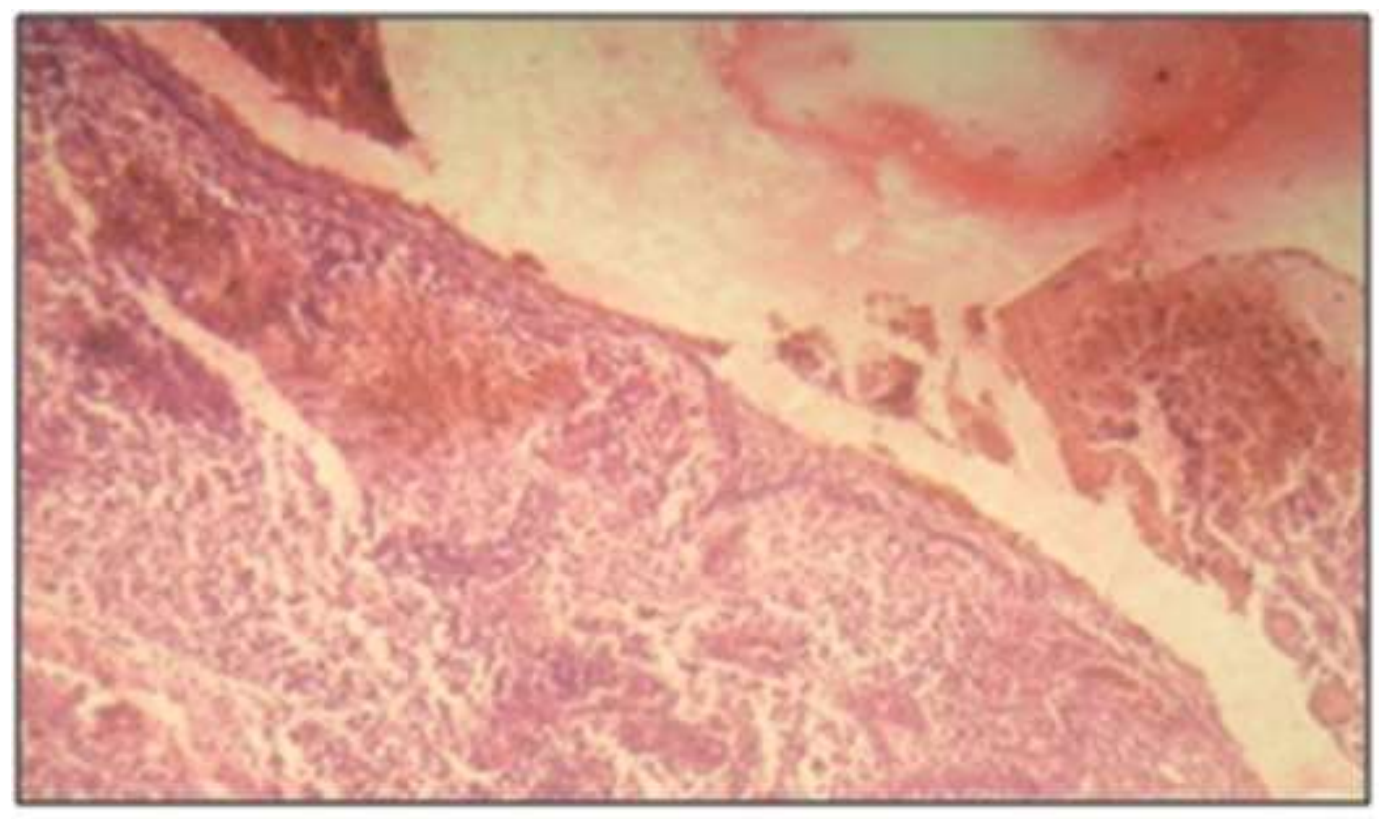

Fig. 5: Dentigerous cyst like change 10X.

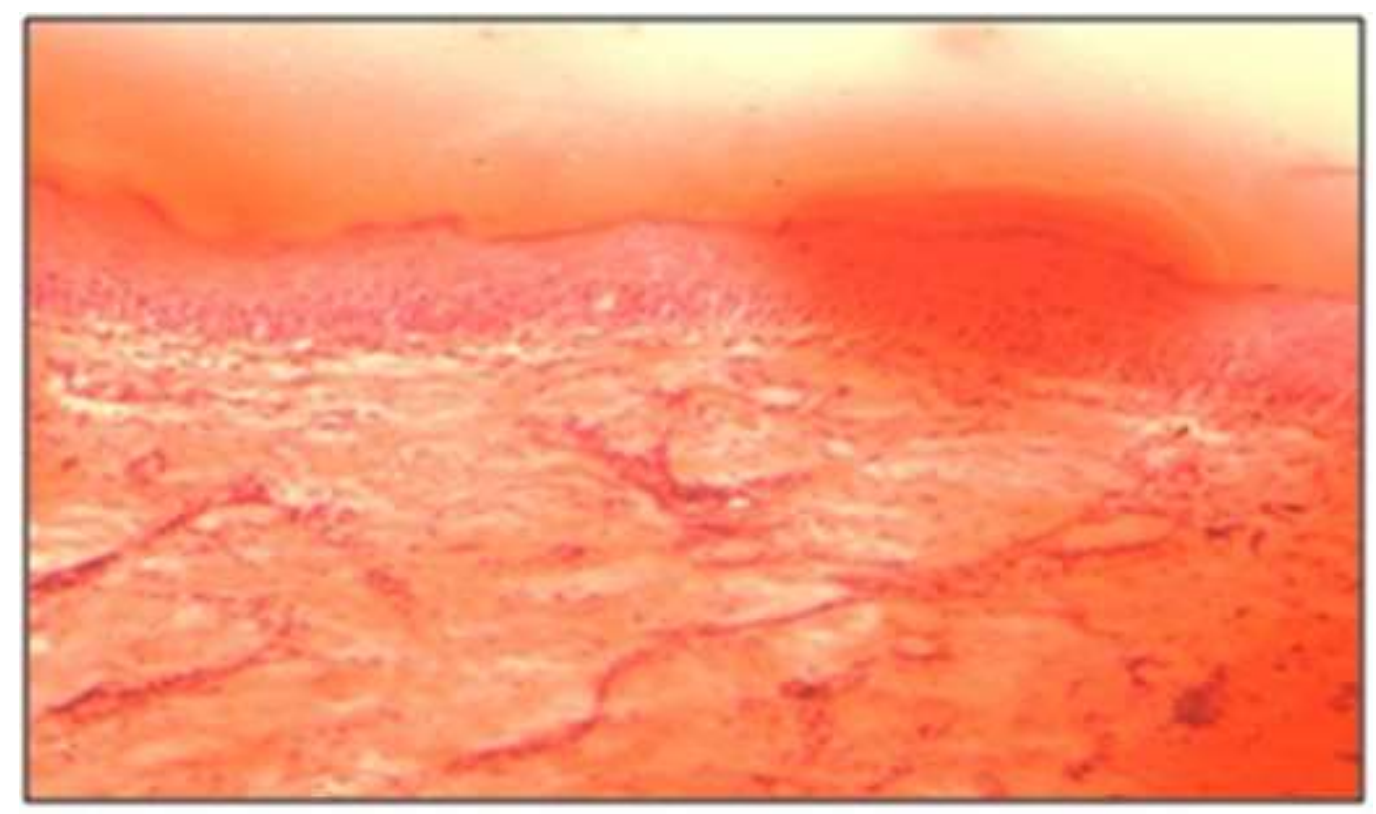

Fig. 6: Odontogenic keratocyst like change 10X. 


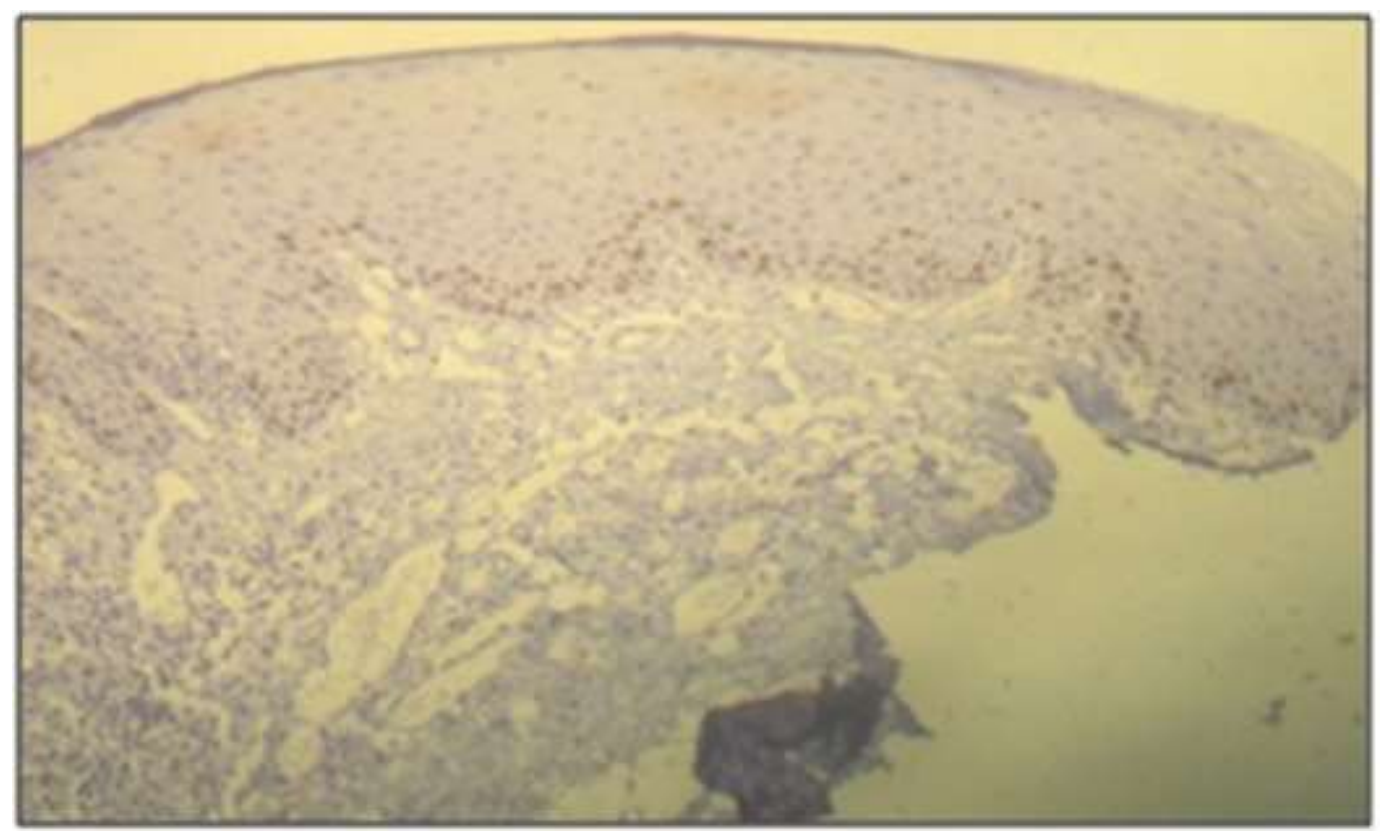

Fig.7: Ki-67 expression in the basal and suprabasal layers 10X. 